\title{
Science's new social contract with society
}

\author{
Michael Gibbons
}

\begin{abstract}
Under the prevailing contract between science and society, science has been expected to produce 'reliable' knowledge, provided merely that it communicates its discoveries to society. A new contract must now ensure that scientific knowledge is 'socially robust', and that its production is seen by society to be both transparent and participative.
\end{abstract}

$\mathrm{M}$ odern science has until recently flourished partly because of a stable, underlying agreement between its practitioners and the rest of society. In other words, there has been a social contract between science and society, an arrangement built on trust which sets out the expectations of the one held by the other, and which - in principle - includes appropriate sanctions if these expectations are not met.

This social contract has been made up of several individual elements, reflecting broader contracts between government and society, between industry and society, and between higher education and society. The contract between university science and society, for example, has been based traditionally on the understanding that universities will provide research and teaching in return for public funding and a relatively high degree of institutional autonomy; under this contract, the universities, often supported through research-funding agencies, have been expected to generate fundamental knowledge for society, and to train the highly qualified manpower required by an advanced industrial society.

By contrast, the contract with industrial research and development (R\&D) has been based on an understanding that industry would provide for the appliance of science through the work of its laboratories, and thus carry the discoveries of basic science into product and process innovations. In turn, government science was meant to use research establishments to fill the gap between university science and industrial R\&D. The understanding has been that the state has been directly responsible for carrying out research related to national need; for example, in defence, energy, public health and standards.

For most of the twentieth century, universities, government research establishments and industrial laboratories have therefore operated relatively independently, developing their own research practices and modes of behaviour. Recently, however, this relative institutional impermeability has gradually become more porous. Privatization policies,

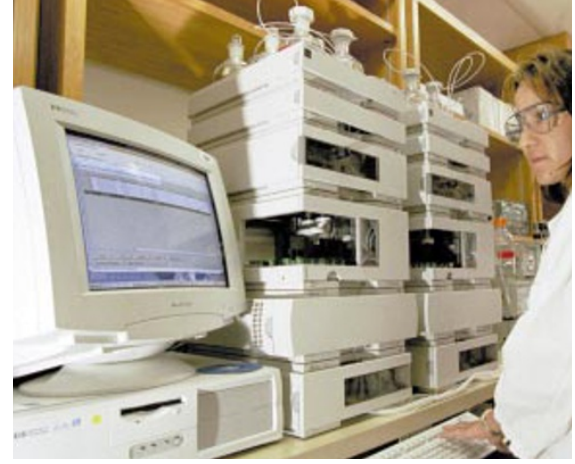

Traditional boundaries between university and industrial science, and between basic and applied research, are disappearing. As a result, science and society are invading each other's domain, requiring a rethinking of previous responsibilities.

for example, have moved many government research establishments into the market place. With the relaxation of the Cold War, governments have shifted their priorities from security and military objectives to maintaining international competitiveness and enhancing the quality of life. And many long-established industries have been denationalized, while in many countries companies previously dependent upon government for $\mathrm{R} \& \mathrm{D}$ support through military technology projects have had to find these resources elsewhere, or in partnership with others, to compete in international markets.

Meanwhile the expansion of higher education has been accompanied by a culture of accountability that has impacted on both teaching and research. In research, many academics have had to accept objective-driven research programmes, whereas research funding agencies have been increasingly transformed from primarily responsive institutions, responsible for maintaining basic science in the universities, into instruments for attaining national technological, economic and social priorities through the funding of research projects and programmes.
These trends can be observed internationally, even if their precise form and timing has varied between countries. Cumulatively, they signal the end of the institutional arrangements through which science flourished during and after the Second World War, and thus mark the expiry of the social contract between science and society that has dominated this period. A new social contract is now required. This cannot be achieved merely by patching up the existing framework. A fresh approach — virtually a complete 'rethinking' of science's relationship with the rest of society - is needed.

Reflecting complexity and diversity One aspect of this new contract is that it needs to reflect the increasing complexity of modern society. For example, there are no longer clear demarcation lines between university science and industrial science, between basic research, applied research and product development, or even between careers in the academic world and in industry. There is now greater movement across institutional boundaries, a blurring of professional identities and a greater diversity of career patterns.

But the price of this increased complexity is a pervasive uncertainty. One way of looking at this is in terms of an erosion of society's stable categorizations, namely the state, market, culture and science. Alternatively, it can be seen as the cumulative effect of parallel evolutionary processes. For there has been a co-evolution in both society and science in terms of the range of organizations with which researchers are prepared to work, the colleagues with whom they collaborate, and topics considered interesting. Whatever viewpoint one takes, science is now produced in more open systems of knowledge production.

One consequence is that the norms and practices of research in university and industrial laboratories have converged. There are still differences between universities and industry, but these do not impact on what is considered sound scientific practice ${ }^{1}$. Indeed, science and society more generally 


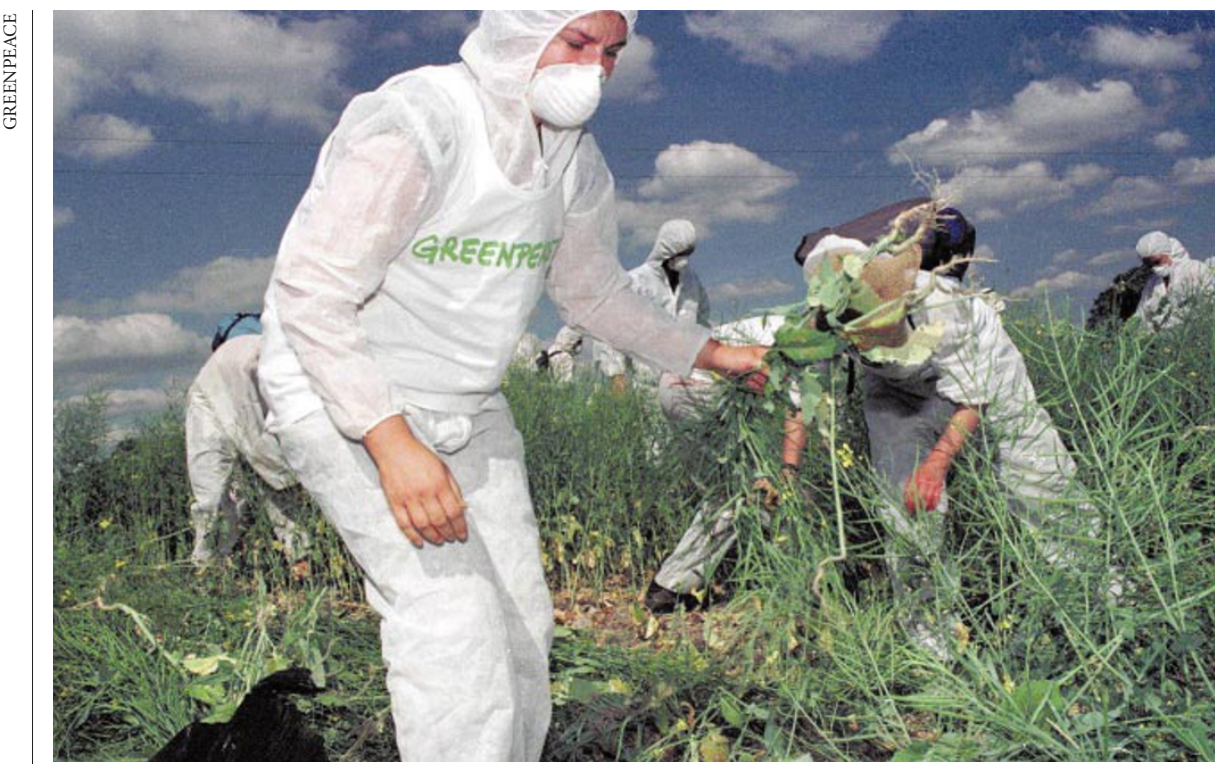

Both pressure groups and ordinary consumers are demanding that the debate surrounding the health implications of GMOs be broadened to include the perspectives of the non-expert community.

have each invaded the other's domain, and the lines demarcating the one from the other have virtually disappeared.

As a result, not only can science speak to society, as it has done so successfully over the past two centuries, but society can now 'speak back' to science. The current contract between science and society was not only premised on a degree of separation between the two, but also assumed that the most important communication was from science to society. Science was seen as the fountainhead of all new knowledge and, as part of the contract, was expected to communicate its discoveries to society. Society in turn did what it could to absorb the message and through other institutions - primarily industry - to transform the results of science into new products and processes.

Science was highly successful working in this mode, and for as long as it delivered the goods, its autonomy was seldom contested. Yet this success has ironically itself been instrumental in changing its relationship with society, drawing science into a larger and a more diverse range of problem areas, many lying outside traditional disciplinary boundaries. It is this increasingly intense involvement of science in society over the past half a century that has created the conditions that underpin the growing complexity and the pervasive uncertainty in which we live, and encouraged the social and behavioural experiments described above.

But if it is widely recognized that science is transforming modern society, it is less often appreciated that society, in speaking back, is transforming science. I will use the term 'contextualization' to describe this process, and 'contextualized knowledge' as the outcome of this reverse communication. Contextualization affects modern science in its organization, division of labour and day-to-day practices, and also in its epistemological core.

In relation to the former, for example, research carried out in both industrial and government laboratories, as well as the funding policies of research-funding agencies, have opened up to a wide range of socioeconomic demands, admitting more and more cross-institutional links, and thus altering the balance between the different sources of funding of academic research. Thus in 'speaking back' to science, society is demanding various innovations, for example the pursuit of national objectives, the contribution to new regulatory regimes and acknowledgement of the multiplication of user-producer interfaces.

In relation to the latter, the epistemological dimension, the increasing importance of 'context' is also reflected in a relatively rapid shift within science from the search for 'truth' to the more pragmatic aim of providing a provisional understanding of the empirical world that 'works'. John Ziman, former physicist and long-time contributor to social studies of science, has described science as a form of 'reliable knowledge' that becomes established not in terms of an abstract notion of objectivity but, concretely, in terms of the replicability of research statements and the formation of a consensus within the relevant peer group ${ }^{3}$. Reliable knowledge is therefore defined as such because it 'works'.

But what 'works' has now acquired a further dimension that can best be described as a shift from 'reliable knowledge' to what Nowotny et al. call 'socially robust' knowledge $^{4}$. The latter characterization is intended to embrace the process of contextualization. For 'socially robust' knowledge has three aspects. First, it is valid not only inside but also outside the laboratory. Second, this validity is achieved through involving an extended group of experts, including lay 'experts'. And third, because 'society' has participated in its genesis, such knowledge is less likely to be contested than that which is merely 'reliable'.

\section{Socially robust knowledge}

My argument is that we are currently witnessing a significant shift from 'reliable' to 'socially robust' knowledge. Three observations can immediately be made. The first is that the basic conditions and processes that have underpinned the production of 'reliable knowledge' are not necessarily compromised by the shift to 'socially robust knowledge'. Indeed, if these conditions and processes have been undermined, it may have been as much by the narrow outlook of much scientific practice as by any attempt to widen the range of stakeholders, or more systematically to take into account the context in which science is produced.

The second observation is that reliable knowledge has always only been reliable within boundaries. Science was recognized as inherently incomplete because it is, primarily, a method rather than a final answer. But to achieve a reasonable degree of reliability, the problem terrain had also to be circumscribed, and judgements on what is included there restricted to those of a peer group, rather than opened to the scientific community as a whole.

Both aspects of reliable knowledge are carried forward into socially robust knowledge. But although knowledge remains incomplete, this is no longer only in the conventional sense that it will eventually be superseded by superior science; rather it means that it may be sharply contested, and no longer remains within the controlled environment of scientific peers. This shift involves renegotiating and reinterpreting boundaries that have been dramatically extended, so that science can no longer not be validated as reliable by conventional discipline-bound norms; while remaining robust, science must now be sensitive to a much wider range of social implications.

An example is the current debate surrounding genetically modified organisms (GMOs). Here, specialist peer groups have been challenged not only by pressure groups but also by ordinary consumers, for whom the research process is far from transparent, and who are demanding that it be more so. Here, knowledge of the health implications of GMOs may be 'reliable' in the conventional scientific sense; but it is not socially robust, and will not become so until the peer group is broadened to take into account the perspectives and concerns of a much wider section of the community. 


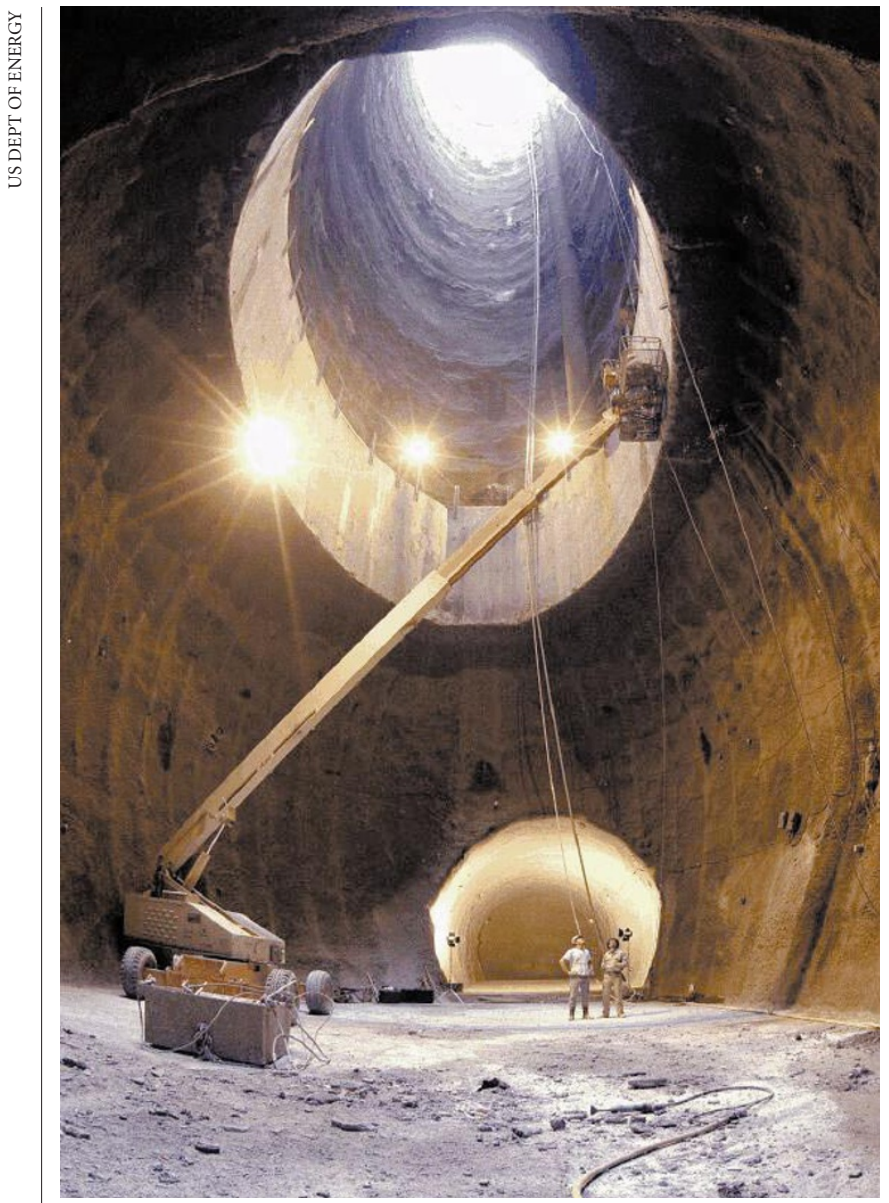

A failure to persuade the broad public of the value of the US Superconducting Super Collider research programme may have contributed to the collapse of funding for the project.

There was also a degree of contestation in the United States about the value of the Superconducting Super Collider (SSC), plans for which were dropped in 1992. In this case, however, unlike the case with GMOs, there was no spontaneous backlash from society generally about the value of the knowledge. Rather, it has been argued that the collapse of funding for the project was a result of the unwillingness (or inability) of a narrow disciplinary group to extend its boundaries sufficiently to persuade other scientists and politicians that the research would be of wide benefit ${ }^{5}$. Again we see a failure to achieve sufficient social robustness in the research process, however reliable it maybe in its own terms.

The third observation is that the epistemological core of science has, over time, become crowded with norms and practices that cannot be reduced easily to a single generic methodology, or, more broadly, to privileged cultures of scientific inquiry. There is no one set of practices that describe, much less lead to, good science. The case for science can still be made in essentially functionalist terms; but many more factors now need to be taken into account before a solution that 'works' can be adopted.

One outcome of all these changes is that the sites at which problems are formulated and negotiated have moved from their previous institutional locations in government, industry and universities into the 'agora' - the public space in which both 'science meets the public', and the public 'speaks back' to science. This is a space in which the media is increasingly active, and in which the new communication technologies play a prominent role. It is also the domain in which contextualization occurs. Neither state nor market, neither exclusively private nor exclusively public, the agora is where today's societal and scientific problems are framed and defined, and their 'solutions' are negotiated.

\section{Narratives of expertise}

The factor that has come to the fore in the agora is the role of scientific and technical expertise that is so crucial to decision making in highly industrialized societies. This role is changing as expertise spreads throughout society, resulting in the fragmentation of established links between expertise and institutional structures, whether of government, industry or the professions. Furthermore, the questions asked of experts are neither the same, nor simple extensions of, the ones that arise in their specialist fields of study. Experts must now extend their knowledge to widely disparate areas, and try to integrate what they 'know' now with what others want to 'do' in the future.

Collective narratives of expertise need to be constructed to deal with the complexity and the uncertainty generated by this fragmentation. Such narratives are challenging to their participants. Experts must respond to issues and questions that are never merely scientific and technical, and must address audiences that never consist only of other experts. The limits of competence of the individual expert call for the involvement of a wide base of expertise that has to be carefully orchestrated if it is to speak in unison.

Since expertise now has to bring together knowledge that is itself distributed, contextualized and heterogeneous, it cannot arise at one specific site, or out of the views of one scientific discipline or group of highly respected researchers. Rather it must emerge from bringing together the many different 'knowledge dimensions' involved. Its authority depends on the way in which such a collective group is linked, often in a self-organized way. Breakdowns in social authority arise when links are inadequately established, as has occurred in European debates over GMOs.

\section{Rethinking science}

These four inter-related processes - coevolution, contextualization, the production of socially robust knowledge and the construction of narratives of expertise form a framework both for rethinking science and for understanding any new social contract between society and science. Co-evolution denotes an open interaction between science and society which generates variety through experimentation, whether in scientific problems, colleagues or institutional designs, with the selective retention of certain choices, modes or solutions. This is so even while these experimental approaches, in responding to uncertainty and complexity, not only promote permeability but also generate more complexity and uncertainty, thus encouraging further experimentation.

Greater permeability provides the basis for increased contextualization by increasing the routes through which society can 'speak back' to science. Denser communication itself brings an imperative to produce socially robust knowledge that is seen as valid not only inside but also outside the walls of the laboratory, in terms of being accepted as legitimate.

As the walls of laboratories have opened up, more and more scientists have taken their places as actors in the agora, broadening the range of experts whose view might be sought on a particular problem or issue. To cope with this, a further development in the use of scientific and technical experts is needed. For reliable knowledge can only become socially robust if society sees the process of knowledge production as transparent and participative. The old image of science working autonomously will no longer suffice. Rather, a reciprocity is required in which not only does the public understand how science 
works but, equally, science understands how its publics work.

The process of rethinking science, now that the line that used to separate science and society has virtually disappeared, has scarcely begun ${ }^{5}$. But several changes in perspective must be initiated before a new social contract can emerge. First, the need for contextualization means that the (unknowable) implications as well as the (planned or predictable) applications of scientific research have to be embraced.

Research activities now transcend the immediate context of application, and begin to reach out, anticipate and engage reflexively with those further entanglements, consequences and impacts that it generates. This 'context of implication' always transcends the immediate 'context of application' in which it occurs. It may embrace neighbouring research fields, and as yet obscurely recognized future uses. Taking the 'context of implication' seriously opens the door to those previously excluded from decisions about research. The individuals now involved may be encountered haphazardly as individuals, perhaps colleagues or rivals. They may come from other scientific disciplines, or from the 'user' community. Whatever their origins, scientific knowledge will increasingly need to be tested not only against nature, but against (and hopefully also with) other people.

Furthermore, while it is important to define problems, and then assemble the intellectual, human and financial resources needed to solve them, this is not, in itself, sufficient to guarantee the reflexivity characteristic of socially distributed knowledge production. In contrast, a process of contextualization that attempts to embrace unpredictable and unintended implications demands reflexivity, as it is intended to incorporate future potential implications into the research process from the very beginning. It thus goes far beyond a conventional 'forward look' or 'technology foresight' exercise.

This has several consequences. One is the need for strategies to 'fix' more accurately the implications of knowledge production. This might be done by identifying areas in which significant implications of particular research projects are likely to arise without being pinpointed exactly, making it necessary to 'prospect' for these (presently unknowable) implications. Such a process might, for example, involve consulting other knowledge producers and users, as well as wider social constituencies, in order to carry out a form of 'triangulation' survey. Perhaps every research proposal and project should include a deliberate strategy for identifying its 'context of implication'. This might best be achieved by including those likely to be implicated perhaps unknowingly — as well as the conscious carriers of social knowledge.
A second need is for the process of contextualization to be internalized. The 'context of application' can be managed through 'external' mechanisms such as 'forward look' and 'technology foresight' exercises, and through science and technology parks and technology transfer or industrial liaison offices within universities. In contrast, the 'context of implication' needs to be internalized by researchers if it is to be effective. It is expressed through routes, often informal, that cannot easily be incorporated into administrative procedures. These new lines of communication need to be encouraged and recognized institutionally. This cannot be done by communications experts - contextualization is not a public relations exerciseor by asking journalists to develop popular accounts of the significance of research.

A further important point is that the more open and comprehensive the scientific community, the more socially robust will be the knowledge it produces. This is contrary to the traditional assumption that there is a strong relationship between the social and intellectual coherence (and, therefore, the boundedness) of a scientific community, and the reliability of the knowledge it produces. Reliable knowledge may have been best produced by such cohesive (and therefore restricted) scientific communities. But socially robust knowledge can only be produced by much more sprawling socio/scientific constituencies with open frontiers.

At the same time, socially robust knowledge is superior to reliable knowledge both because it has been subject to more intensive testing and retesting in many more contexts - which is why it is 'robust' - and also because of its malleability and connective capability. Its context is not predetermined or fixed, but open to ceaseless renegotiation. Instead of achieving a precarious invariance by establishing strict limits within which its truthfulness can be tested, as reliable knowledge does, socially robust knowledge is the product of an intensive (and continuous) interaction between data and other results, between people and environments, between applications and implications.

It is also clear that science must leave the ivory tower and enter the agora. To increase the effectiveness with which the agora operates, the self-organizing capacity of all participants needs to be enhanced. Here, there is tension between the desire for individual or institutional autonomy and the increasing demands for accountability on both individuals and institutions.

Increasing the capacity for self-organizing means that participants need to act more reflexively. But one cannot enhance responsiveness by simply increasing the demand for public accountability, as this could make participants more, rather than less, defensive. On the contrary, what is needed is to encourage participants voluntarily to internalize accountability. Indeed there is an analogy between the relationships between autonomy and self-organization on the one hand, and between reliable and socially robust knowledge on the other. In the agora, the conditions that promote greater selforganization also promote the generation of socially robust knowledge.

\section{Conclusion}

To summarize, I have argued in this paper that the prevailing contract between science and society was set up to sustain the production of 'reliable knowledge'; a new one must ensure the production of 'socially robust knowledge'. The prevailing contract is governed by the rules of bureaucratic rationality, with society linked to 'people' primarily through representative institutions. A new contract will require more open, socially distributed, self-organizing systems of knowledge production that generate their own accountability and audit systems. Under the prevailing contract, science was left to make discoveries and then make them available to society. A new contract will be based upon the joint production of knowledge by society and science.

A new social contract will therefore involve a dynamic process in which the authority of science will need to be legitimated again and again. To maintain this, science must enter the agora and participate fully in the production of socially robust knowledge. According to some observers, we can already see this approach emerging in the management of large technology projects. Thomas $\mathrm{P}$. Hughes, the eminent American historian of technology, has identified a new ethos among engineers who now recognize that the deeper involvement of communities in decision making actually produced better engineering solutions in a number of projects ${ }^{6}$. If the boundaries between science, technology and society are becoming more permeable, why should not a similar approach in science likewise produce more socially robust solutions?

Michael Gibbons, a former director of the Science Policy Research Unit at the University of Sussex, is now secretary-general of the Association of Commonwealth Universities, 36 Gordon Square, London WC1H OPF, UK.

1. van Duinen, R. J. European research councils and the Triple Helix. Sci. Public Policy 25, 381-386 (1998).

2. Daston, L. \& Galison, P. The image of objectivity. Representations 40, 81-128 (1992).

3. Ziman, J. Reliable Knowledge Canto Edn (Cambridge Univ. Press, Cambridge, 1991)

4. Nowotny, H., Scott, P. \& Gibbons, M. Re-thinking Science: Knowledge Production in an Age of Uncertainty (in the press).

5. Gieryn, T. F. in Handbook of Science and Technology Studies (eds Jasanoff, S. et al.) 393-443 (Sage Publishers, London, 1995). 6. Hughes, T. P. Rescuing Prometheus 301-303 (Pantheon Books, New York, 1998).

Acknowledgements. The research described in this essay is taken from a report entitled 'Re-thinking science: knowledge production in a Mode 2 Society', prepared by H. Nowotny, P. Scott and M. Gibbons for, and funded by, the Tercentenary Fund of the Royal Swedish Bank and the Swedish Council for Higher Education. 